\title{
Propafenone is not effective for severe ventricular arrhythmias in Andersen-Tawil syndrome
}

Piotr Bienias ${ }^{1}$, Anna Kostera-Pruszczyk², Maria Miszczak-Knecht ${ }^{3,4}$, Michał Ciurzyński ${ }^{1}$, Piotr Pruszczyk ${ }^{1}$

${ }^{1}$ Department of Internal Medicine and Cardiology, Medical University of Warsaw,
Warsaw, Poland
${ }^{2}$ Department of Neurology, Medical University of Warsaw, Warsaw, Poland
${ }^{3}$ Department of Pediatric Cardiology and General Pediatrics, Medical University
of Warsaw, Warsaw, Poland
${ }^{4}$ Department of Cardiology, Children's Memorial Health Institute, Warsaw, Poland

Submitted: 23 July 2015

Accepted: 3 November 2015

Arch Med Sci 2018; 14, 1: 248-250

DOI: 10.5114 /aoms.2016.61010

Copyright (c) 2016 Termedia \& Banach

Andersen-Tawil syndrome (ATS), which is sometimes named long QT syndrome type 7 , is a rare genetic potassium channelopathy combining frequent and sometimes serious ventricular arrhythmias (VA), a prominent U-wave in electrocardiography, periodic paralysis and various dysmorphic features. In most ATS patients a mutation in the KCN/2 gene was found (encoding potassium channel Kir2.1) [1-3]. Since ATS is a rare disease, no evidence-based recommendations for VA management are available either. Successful $\beta$-blocker, calcium channel blocker or flecainide medication has been described in many cases [1, 2, 4-6]. However, limited data on propafenone treatment and its efficacy in ATS are available [7].

We present an 18-year-old woman with genetically confirmed ATS (G146R mutation of KCJN2 gene) in whom severe VA were observed since her childhood. She presented typical ATS dysmorphic features (hypertelorism, mandibular hypoplasia and syndactyly) and suffered from periodic paralysis. Andersen-Tawil syndrome was also diagnosed in her mother (the same mutation), but she presented only slight dysmorphic features and no VA. The patient's father and her two brothers were healthy. At presentation the patient's echocardiography was normal. In many Holter recordings asymptomatic typical VA were observed including frequent pairs, bigeminy or trigeminy and non-sustained bidirectional ventricular tachycardia (BVT), up to 50-60 thousand ventricular beats daily. After VA diagnosis, various antiarrhythmic drugs were prescribed and finally flecainide was found to be the most effective, with significant VA reduction to 5-10 thousand beats daily and 5-20 short BVT. Due to logistic problems with flecainide supply in our country, we decided to replace flecainide with readily available propafenone, which is also a Vaughan-Williams class IC antiarrhythmic agent and is registered for VA treatment. After admission to our department the patient received propafenone $150 \mathrm{mg}$ t.i.d. and metoprolol succinate $50 \mathrm{mg}$ daily with a small increase of VA in initial clinical observation. Unfortunately, subsequent Holter recordings showed stable intensified VA (15-20 thousand beats daily), and a substantial increase of the number, length and heart rate of BVT (400-800 episodes daily, up to 20-25 s). Typical BVT and standard ECG of our patient are presented in the Figure 1. Moreover,

\author{
Corresponding author: \\ Piotr Bienias \\ Department \\ of Internal Medicine \\ and Cardiology \\ Medical University \\ of Warsaw \\ 4 Lindleya St \\ 02-005 Warsaw, Poland \\ Phone: +48 604405365 \\ E-mail: pbienias@mp.pl
}




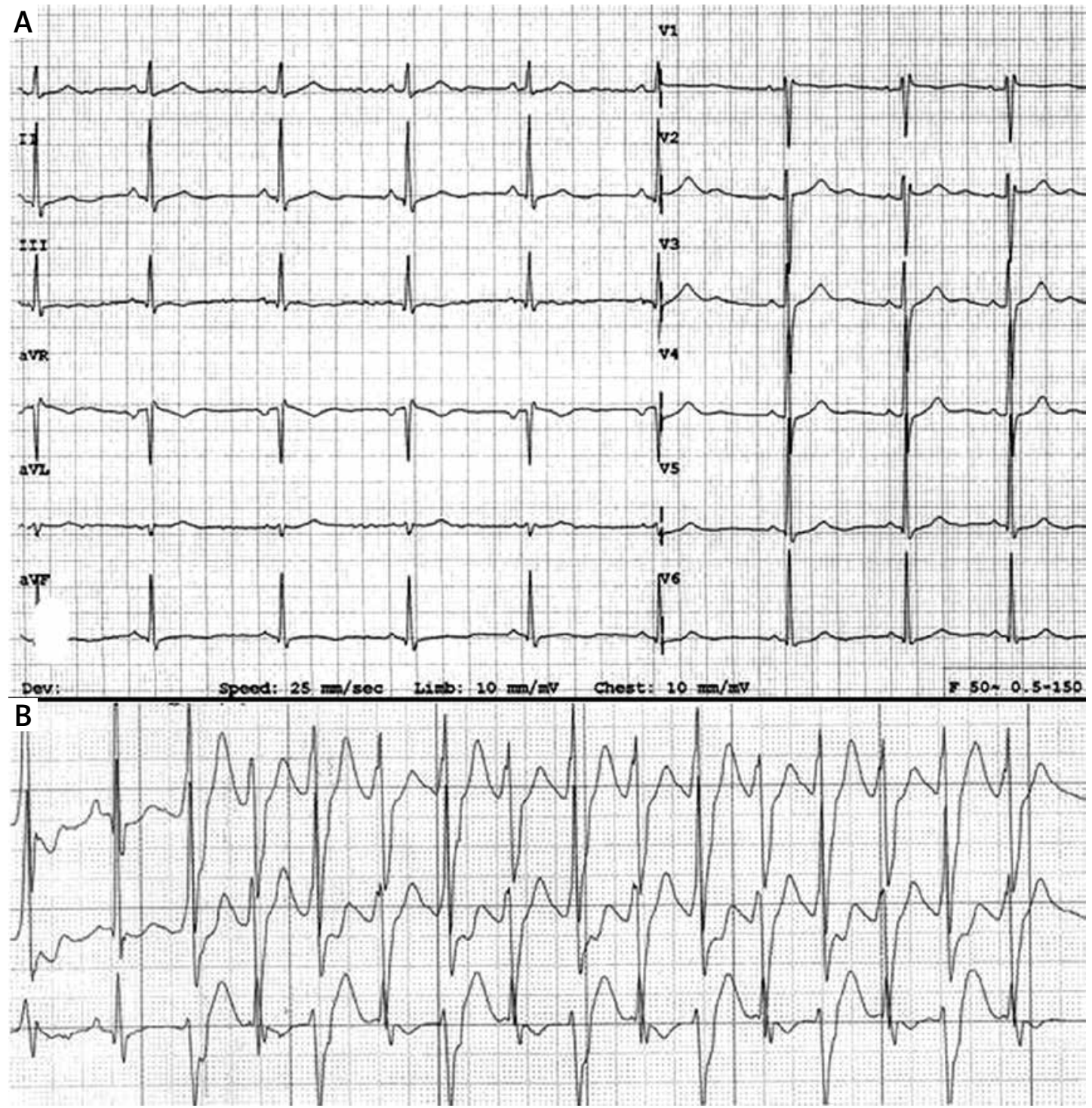

Figure 1. A - Standard ECG. Sinus rhythm 64 bpm. QT/QTc intervals 430/450 ms. Distinct U wave in leads V2-V3.

B - 3-channel 24-hour Holter monitoring. Nonsustained bidirectional ventricular tachycardia (BVT)

the patient became pregnant with further exacerbation of VA (especially BVT), so we decided to reintroduce flecainide treatment $100 \mathrm{mg}$ b.i.d., with dramatic VA reduction ( 6 thousand beats, 40 very short BVT only). After propafenone cessation for 2 days the patient received only $\beta$-blocker and further intensity of VA with 1300 episodes of BVT was observed then. It is indirect evidence that propafenone did not exert a proarrhythmic effect, but it lacked sufficient efficacy in our patient.

Various genetic factors (over 40 mutations and 3 deletions of the $K C N J 2$ gene) and also extremely low prevalence of ATS (approx. 1 per 1 million population) cause that knowledge of this disease is based on observations of small groups of patients and case reports only. The present woman is one of 15 genetically confirmed ATS subjects who have been observed and treated in our departments [3]. Serious and severe VA (especially
BVT and torsades de pointes leading to ventricular fibrillation) may be a cause of sudden cardiac death (SCD). However, the risk of SCD in all ATS patients is relatively small, and most often it is estimated at below $3-10 \%$ of cases $[1,2,8]$. Successful antiarrhythmic treatment in our patient was important not only due to SCD risk, but also due to a temporary decrease of left ventricular ejection fraction during intensified VA, which was observed in her childhood. As ATS is a rare disease, no definitive recommendations for VA management are available. It seems that $\beta$-blocker treatment is of clinical value, but successful calcium channel blocker medication and a combination of flecainide plus $\beta$-blockers have also been described [1, 2, 4, 5]. Unfortunately, pharmacological treatment is not beneficial in many patients. In some ATS cases implantation of a cardioverter-defibrillator is indicated, but it is controversial [8]. 
Similarly to other channelopathies, ablation at tempts fail in many cases.

We tried to treat our patient with propafenone because it is a similar agent to flecainide - similar, but not the same. In contrast to flecainide, propafenone has additional clinically significant $\beta$-blocker activity. Prolonged action potential duration and efficacy of standard propafenone treatment may also depend on metabolic variability among consecutive subjects (fast and slow metabolizers). On the other hand, flecainide additionally demonstrates a minor calcium channel blockade effect, and it has affinity for the open state sodium channel only [9-11]. We did not assess concentrations of propafenone and its active metabolites. However, our patient was a rather slim woman (body mass approx. $50 \mathrm{~kg}$ ), and we were afraid to prescribe larger doses than $450 \mathrm{mg}$ daily. We do not conclude that propafenone will not be effective in all ATS patients either. It is worth noting that some KCN/2 mutations are associated with extremely high risk of severe VA [12]. Maybe a type of mutation influences the response to the prescribed type of drug too?

In conclusion, we present the first case of ATS in a patient with severe and potentially life-threatening VA who was treated with propafenone. However, this therapy was ineffective, and flecainide had to be reintroduced, with substantial improvement.

\section{Conflict of interest}

The authors declare no conflict of interest.

\section{References}

1. Wilde AA. Andersen-Tawil syndrome, scarier for the doctor than for the patient? Who, when, and how to treat. Europace 2013; 15: 1690-2.

2. Nguyen HL, Pieper GH, Wilders R. Andersen-Tawil syndrome: clinical and molecular aspects. Int J Cardiol 2013; 170: 1-16.

3. Kostera-Pruszczyk A, Potulska-Chromik A, Pruszczyk P, et al. Andersen-Tawil syndrome: report of 3 novel mutations and high risk of symptomatic cardiac involvement. Muscle Nerve 2015; 51: 192-6.

4. Miyamoto K, Aiba T, Kimura H, et al. Efficacy and safety of flecainide for ventricular arrhythmias in patients with Andersen-Tawil syndrome with KCNJ2 mutations. Heart Rhythm 2015; 12: 596-60.

5. Hayashi H, Kawaguchi T, Horie M. Effect of flecainide on T-wave alternans in Andersen-Tawil syndrome. Ann Noninvasive Electrocardiol 2014; 19: 383-6.

6. Pellizzón OA, Kalaizich L, Ptácek LJ, Tristani-Firouzi M, Gonzalez MD. Flecainide suppresses bidirectional ventricular tachycardia and reverses tachycardia-induced cardiomyopathy in Andersen-Tawil syndrome. J Cardiovasc Electrophysiol 2008; 19: 95-7.

7. Junker J, Haverkamp W, Schulze-Bahr E, Eckardt L, Paulus W, Kiefer R. Amiodarone and acetazolamide for the treatment of genetically confirmed severe Andersen syndrome. Neurology 2002; 59: 466.
8. Bienias P, Kostera-Pruszczyk A, Bieganowska K, Miszczak-Knecht M, Pruszczyk P. Should a cardioverter-defibrillator be implanted in an Andersen-Tawil syndrome patient with severe ventricular arrhythmias and syncope? Kardiol Pol 2014; 72: 755.

9. Roden DM. Antiarrhythmic drugs. In: Goodman and Gilman's The Pharmacological Basic of Therapeutics. $11^{\text {th }}$ ed. Brunton LL, Lazo JS, Parker KL (eds). The McGraw-Hill Companies, Inc. 2006; 899-932.

10. Gómez R, Caballero R, Barana A, et al. Structural basis of drugs that increase cardiac inward rectifier Kir2.1 currents. Cardiovasc Res 2014; 104: 337-46.

11. Janson CM, Poelzing S, Shah MJ. Combined inhibition of $\mathrm{Na}^{+}$and $\mathrm{Ca}^{2+}$ channels: a novel paradigm for the treatment of incessant ventricular arrhythmias in Andersen-Tawil syndrome. Heart Rhythm 2014; 11: 318-20.

12. Fernlund E, Lundin C, Hertervig E, Kongstad O, Alders M, Platonov P. Novel mutation in the KCNJ2 gene is associated with a malignant arrhythmic phenotype of Andersen-Tawil syndrome. Ann Noninvasive Electrocardiol 2013; 18: 471-8. 